\title{
Surface tension controls the hydraulic fracture of adhesive interfaces bridged by molecular bonds
}

\author{
Dimitri Kaurin ${ }^{1, *}$ and Marino Arroyo ${ }^{1,2, \dagger}$ \\ ${ }^{1}$ Universitat Politècnica de Catalunya-BarcelonaTech, 08034 Barcelona, Spain. \\ ${ }^{2}$ Institute for Bioengineering of Catalonia, The Barcelona Institute of Science and Technology, 08028 Barcelona, Spain
}

(Dated: November 4, 2019)

\begin{abstract}
Biological function requires cell-cell adhesions to tune their cohesiveness, for instance during the opening of new fluid-filled cavities under hydraulic pressure. To understand the physical mechanisms supporting this adaptability, we develop a stochastic model for the hydraulic fracture of adhesive interfaces bridged by molecular bonds. We find that surface tension strongly enhances the stability of these interfaces by controlling flaw sensitivity, lifetime and optimal architecture in terms of bond clustering. We also show that bond mobility embrittles adhesions and changes the mechanism of decohesion. Our study provides a mechanistic background to understand the biological regulation of cell-cell cohesion and fracture.
\end{abstract}

Cell-cell adhesions are remarkably versatile and tunable interfaces. They need to be strong enough to maintain barrier integrity and cohesion in epithelia under stretch [1, 2], but also need to disengage and reform during morphogenesis $[3,4]$. To accomplish this, they rely on weak, mobile and force-sensitives molecular bonds [5], which enable remodeling yet, collectively, can become strong. However, the physical rules by which such interfaces adapt to the changing requirements imposed by biological function remain poorly understood. Beyond biology, adhesion by low-affinity molecular bonds in reconstituted systems have the potential to achieve the degree of versatility of biological adhesion [6-10].

In important physiological situations, cell-cell junctions are disengaged as a result of hydraulic pressure in the interstitial fluid, which pulls apart neighboring cell membranes. For instance, increased pressure in the extra-cellular medium, caused by poroelasticity or by active ionic transport, can lead to hydraulic fracture of cell-cell and cell-matrix adhesions $[2,11,12]$. Hydraulic disengagement of cell-cell junctions is also required during the opening of fluid-filled luminal cavities (luminogenesis) [13-15]. Despite this seeming generality, the physical mechanisms that control the hydraulic fracture of adhesive interfaces bridged by molecular bonds have not been examined. Here, by studying a simple theoretical model, we identify an unanticipated strong role of surface tension on the stability of such adhesive interfaces.

To understand the fracture physics of cell-cell adhesion, molecular interfaces have been idealized as a collection of stochastic molecular bonds. Previous studies have examined the strength and dynamics interfaces bridged by stochastic bonds [17-19], possibly accounting for the non-uniform force distribution around crack-like defects transmitted by an elastic or viscoelastic medium [20-22]. Here, to examine hydraulically loaded adhesive junctions, we consider a minimal model consisting of a 1D interface of identical stochastic bonds bridging a pressurized cleft and attached to a tense membrane (a tense line in our model), which represents the plasma membrane and/or the thin actin cortex, Fig. 1. Since the membrane is tense under the cleft pressure, we ignore membrane fluctuations, which can however play an important role during adhesion expansion of flaccid vesicles [18]. We further assume that membrane tension is high enough so that the bending rigidity can be neglected in a capillary approxima-

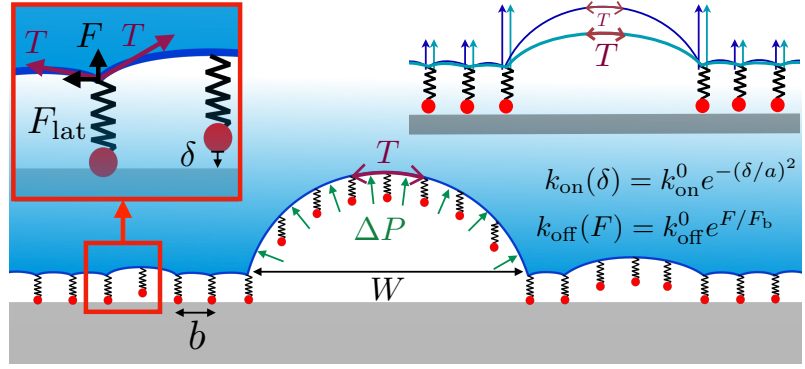

FIG. 1. Adhesive interfaces formed by a tense membrane (here, a line with tension $T$ ) bridged by molecular bonds and enclosing a cavity at pressure $P$. Open bonds can stochastically attach depending on the separation $\delta$ to form a stochastic slip bond, whose off-rate depends exponentially on force according to Bell's model [16]. The system is periodic. Following Laplace's law, each line segment between two neighboring closed bonds is an arc of circle or radius $R=T / P$. This allows us to compute the force on each closed bond $F$ and the distance between opposite potential partners $\delta$, which in turn determine the unbinding and binding rates $k_{\text {off }}$ and $k_{\text {on }}$. We denote by $b$ the spacing between bonds and by $W$ the size of a selected crack. In this model, increasing tension flattens the membrane over the crack, favoring rebinding, but does not affect the force on the closed bonds (top-right inset).

tion, and that it remains constant during bond dynamics [23]. See [24] for further details and discussions. Under these simplifying assumptions and for any open/closed bond configuration, force balance allows us to compute the force $F$ borne by each closed bond, and hence its unbinding rate following Bell's model for a slip bond with force sensitivity $F_{\mathrm{b}}$ [16]. Likewise, from simple geometry we compute the separation between potential partners $\delta$, and hence the binding rate of each open bond [25]. To follow the stochastic dynamics of the model, we use Gillespie's first-reaction method [26].

In our simulations, we chose parameters close to those of integrin/fibronectin bonds in focal adhesions, with $b=32 \mathrm{~nm}$, a force sensitivity of $F_{\mathrm{b}}=4 \mathrm{pN}$, a bond stiffness of $k_{0}=$ $2.5 \cdot 10^{-4} \mathrm{~N} \cdot \mathrm{m}^{-1}$, leading to $a=\sqrt{2 /\left(\beta k_{0}\right)} \approx 6 \mathrm{~nm}$ with $1 / \beta$ the Boltzmann constant times absolute temperature, an unbinding rate in the absence of force of $k_{\mathrm{off}}^{0}=1 \mathrm{~s}^{-1}$, and a ratio between the binding rate at zero separation and the unbinding rate at zero force of $k_{\mathrm{on}}^{0} / k_{\mathrm{off}}^{0}=3$. However, these parameters can change widely depending on the bonds and the 


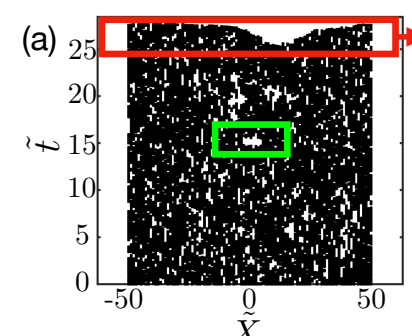

(d)
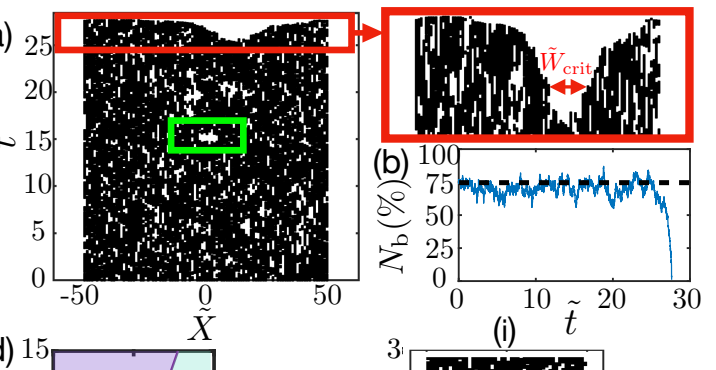

(i) 10
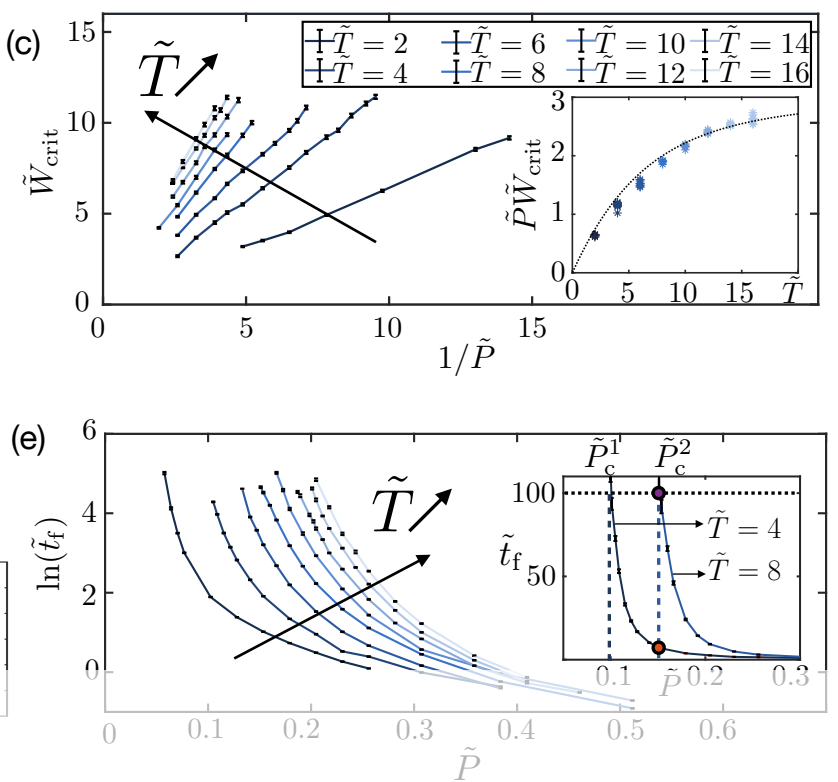

FIG. 2. (a) Kymograph of a representative stochastic simulation, where closed bonds are marked in black and open bonds in white, for $\tilde{T}=4$, $\tilde{P}=0.1$. (b) Corresponding time-evolution of number of closed bonds $N_{\mathrm{b}}$. (c) Critical crack size $\tilde{W}_{\text {crit }}$ as a function of the inverse pressure $1 / \tilde{P}$ for different tensions $\tilde{T}$. Error bars throughout the paper indicate the standard error of the mean (SEM), here for 2000 realizations. By plotting $\tilde{P} \tilde{W}_{\text {crit }}$ as a function of $\tilde{T}$, each of the curves collapses to a point (inset), where the dotted line is a theoretical prediction. (d) Kymographs describing trajectories of cracks at pressure $\tilde{P}=1 / 8$ and initial crack-width $\tilde{W}_{\mathrm{i}}$ indicated by dots in the $(1 / \tilde{P}, \tilde{W})$ plane, where the red line

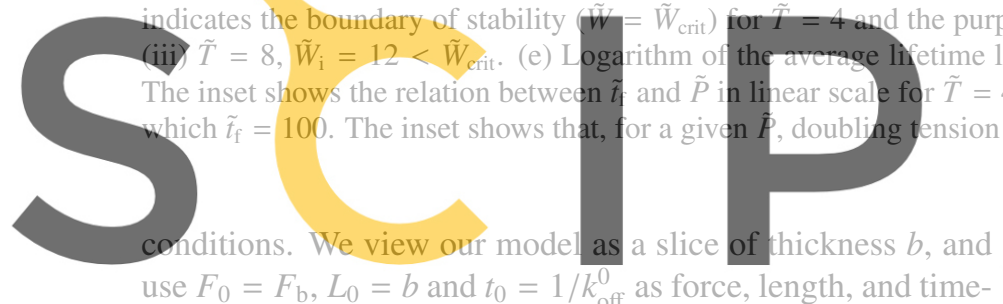

scales to define non-dimensional quantities, e.g. $\tilde{T}=T L_{0} / F_{0}$, Register=fpr freenat https //WwW.scipedia.com to

We first considered a tense adhesive interface containing 100 adhesive molecules in a regular lattice, subjected it to an interstitial pressure, and followed the dynamics, see Fig. 2(a,b). The interface remained cohesive for a long time, during which small cracks of various sizes stochastically opened and closed (see green inset) and the fraction of closed bonds fluctuated around $N_{\mathrm{b}}^{0}=k_{\mathrm{on}}^{0} /\left(k_{\text {on }}^{0}+k_{\text {off }}^{0}\right)$, consistent with low bond forces and small separation distances. At some point, a nascent crack appeared to stochastically grow, and then quickly propagated by sequential breaking of bonds at its margins up to total rupture of the interface. Examination of many trajectories showed that this behavior was general, and interestingly, suggested the notion of a critical crack size $W_{\text {crit }}$ delimiting healing or brutal propagation in analogy with Griffith's classical theory of fracture [27]. However, fracture physics in our model does not result from a thermodynamic competition between elastic and surface energy as in Griffith's theory, but rather on the kinetics of molecular bonds.

To understand the physics determining $W_{\text {crit }}$ and noting that crack growth proceeded by sequential bond breaking at the crack tip, we examined the competition between binding/unbinding of marginal open/closed bonds. To further support the idea of a small process zone near the crack tip, we

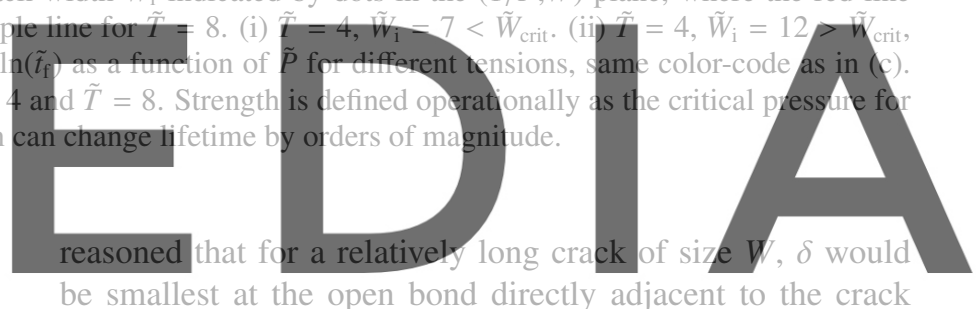

be smallest at the open bond directly adjacent to the crack tip, Fig. 1, and thus crack healing, should be pverwhelmingly

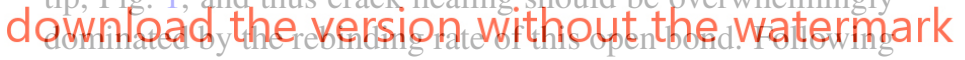

a simple geometric estimation for $\delta$ capturing the flattening of the membrane deflection over a crack as tension increases [24], this rate takes the form [25, 28]

$$
k_{\mathrm{on}} \approx k_{\mathrm{on}}^{0} \exp \left[-\left(\frac{b}{a}\right)^{2} \frac{1}{\left(\frac{2 T}{W P}\right)^{2}-1}\right] .
$$

Similarly, the largest force being borne by the bond defining the crack tip, crack growth should be determined by the unbinding rate of this bond. At the margin of a large enough crack of size $W$, force is tension-independent and can be approximated by $F \approx W P / 2$ [24]. We thus estimated this rate as

$$
k_{\text {off }}=k_{\text {off }}^{0} \exp \left(\frac{W P}{2 F_{\mathrm{b}}}\right) .
$$

The threshold between propagation and healing is characterized by an equal probability of bond breaking and formation at the crack margin, $k_{\text {on }}=k_{\text {off }}$. Examination of Eqs. $(1,2)$ shows that $k_{\text {on }}$ is a monotonically decreasing function of $W$, whereas $k_{\text {off }}$ is monotonically increasing in $W$, and thus $k_{\text {on }}=k_{\text {off }}$ defines a critical length $W_{\text {crit }}$ below which cracks should predominantly heal and above which cracks should grow and become increasingly unstable. Furthermore, $W$ appears grouped 
with $P$ in both expressions, and thus $W_{\text {crit }}$ should be proportional to $1 / P$. Finally, we note that $k_{\mathrm{on}}$ is an increasing function of $T$ since tension brings opposing open bond closer together, but $k_{\text {off }}$ is independent of $T$, and thus we predict that increasing tension should promote crack healing.

To test this theory, we performed systematic simulations for a periodic system with 50 potential bonds initially at quasiequilibrium with $N_{\mathrm{b}}^{0}=k_{\mathrm{on}}^{0} /\left(k_{\mathrm{on}}^{0}+k_{\mathrm{off}}^{0}\right)$ closed bonds. We considered 60 combinations of $T$ and $P$, and for each condition we performed 2000 simulations to estimate $W_{\text {crit }}$ as described in [24]. Our stochastic simulations confirmed the prediction that $W_{\text {crit }}$ should scale linearly with $1 / P$ for each value of $T$, Fig. 2(c). Furthermore, these results are consistent with the prediction that larger tension should lead to larger $W_{\text {crit }}$. Further confirming the simple physical picture provided by Eqs. $(1,2)$, when we plotted $P W_{\text {crit }}$ as a function of $T$, we found that each of these curves collapsed to a point, and that these points followed a relation given implicitly by the condition $k_{\text {on }}=\alpha k_{\text {off }}$, inset in Fig. 2(c), where $\alpha=1.5$ for quantitative agreement, see [24] for a discussion. Importantly, additional calculations considering a 2D membrane enclosing a volumetric cleft and bridged by a 2D arrangement of bonds supported the generality of the physics behind Eqs. $(1,2)$ and of the results in Fig. 2.

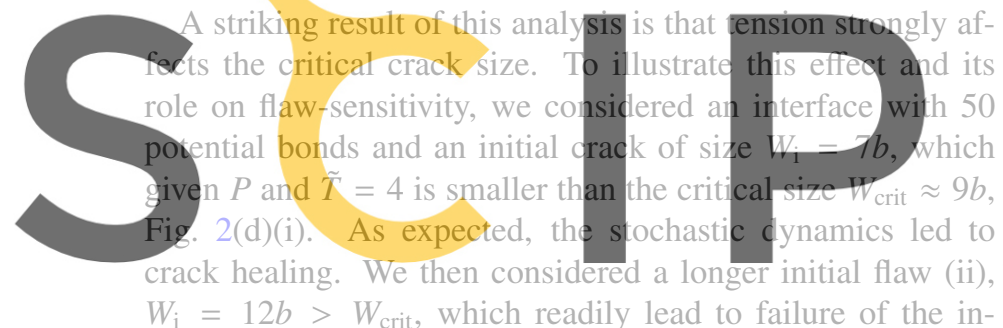

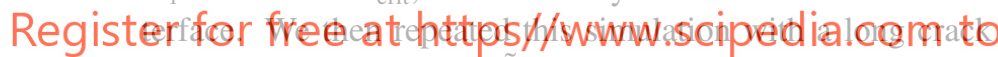
$\left(W_{\mathrm{i}}=12 b\right)$ but doubling $\tilde{T}$, (iii). Because increasing tension increased $W_{\text {crit }}$, in this case much above the initial flaw size, we found that the longer crack now rapidly healed. These results thus show that adhesive interfaces under hydraulic loading can tune their sensitivity to flaws, and thus their ability to disengage or remain cohesive, by controlling surface tension.

Our simulations also provided information about the lifetime of the interface. Although lifetime should depend on the probability of nucleating a crack longer than $W_{\text {crit }}$, and thus on system size, we can still examine its systematic dependence on tension and pressure. Fig. 2(e) shows that, for a given tension, lifetime very rapidly increases as pressure decreases. This abrupt behavior allowed us to define a critical pressure, below which the system remains stable for very long times relative to $t_{0}$ and above which it rapidly fails (inset). Our results show that lifetime is very sensitive to tension, particularly around the critical pressure (see inset), with modest changes in tension leading to changes in lifetime by orders of magnitud. These results further emphasize how adhesion can be tuned by tension.

We then wondered about the effect of diffusion, since molecular bonds can laterally move in the fluid lipid membrane or in the actomyosin cortex as it undergoes turnover [29]. We developed a version of the model where molecules (a)

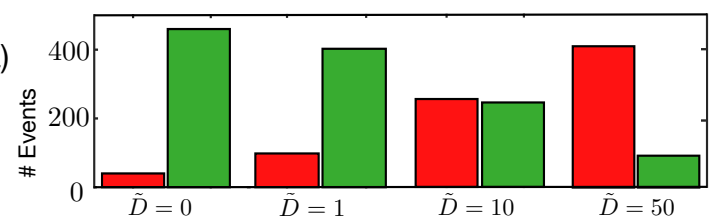

(b)

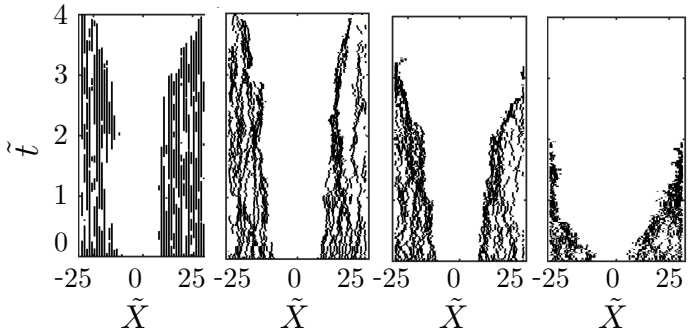

(c)
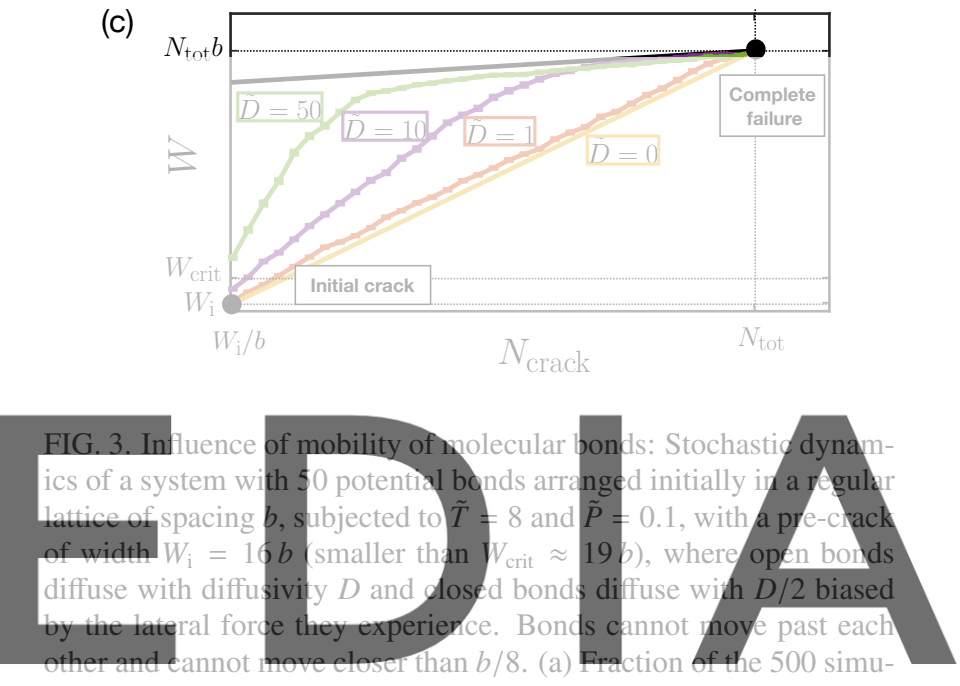

lations leading to failure (red) or healing (green) after a period of 10

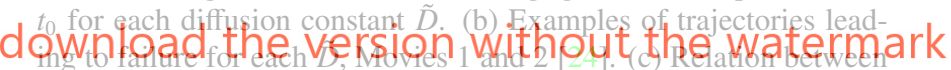

crack length $W$ and the number of open bonds at the crack $N_{\text {crack }}$, obtained by averaging 50 failure trajectories, for different diffusivities. For fixed molecules $(\tilde{D}=0)$ the relation is linear with slope $b$, whereas for large diffusivity, crack length initially grows by motion of bonds at nearly constant $N_{\text {crack }}$ leading to a concentrated adhesion patch, and then by progressive bond breaking with slope $b / 8$ (black line), indicative of a maximal bond packing.

not only react but also diffuse and experience steric effects when crowded. Closed bonds also experience a bias due to their lateral force and, being attached, have smaller diffusivity $D / 2$ compared to that of open bonds $D$ [24]. Since closed bonds at the crack tip experience the largest lateral force pushing them away from the crack, Fig. 1, now cracks could in principle grow by motion of closed bonds. To examine the competition between reaction kinetics and diffusion of bonds [30, 31], we followed the stochastic dynamics of a system with a pre-crack smaller than the critical crack length (strictly valid only for the case of immobile bonds), and tracked the fraction of simulations that reached complete failure after $10 t_{0}$ for different diffusivities, Fig. 3(a). Since crack length is smaller than $W_{\text {crit }}$, for $D=0$ most trajectories lead to healing. Failing realizations proceeding by progressive bond breaking 

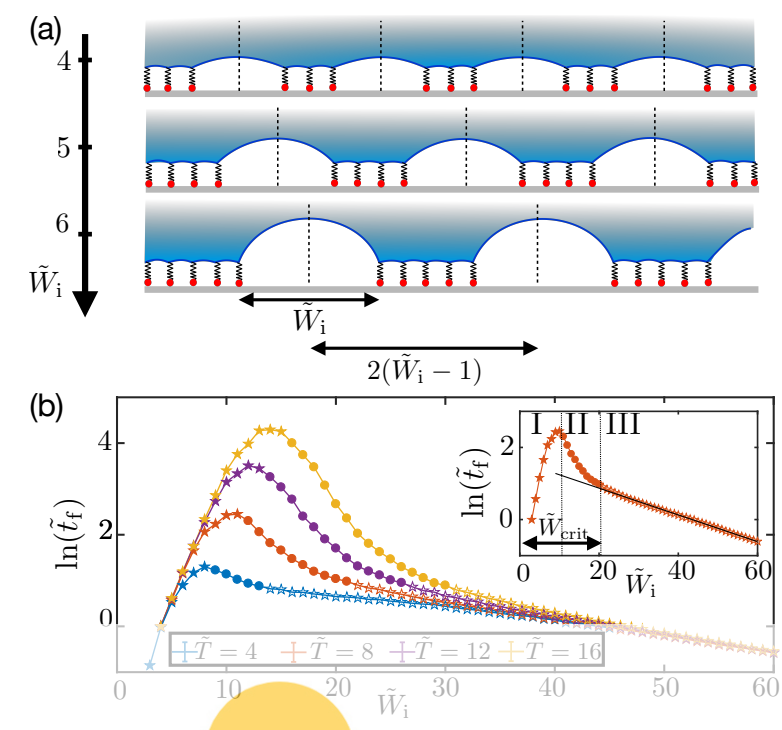

FIG. 4. (a) Sequence of architectures of an adhesive interface with increasing cluster size and cluster separation, $\tilde{W}_{\mathrm{i}}$, but constant bond separation and average density. (b) Logarithm of lifetime $\tilde{t}_{\mathrm{f}}$ of a cluster as a function of $\tilde{W}_{\mathrm{i}}$ for different tensions $\tilde{T}$ and for $\tilde{P}=0.1$ (average lifetimes obtained from 500 simulations). Optimal cluster size is well approximated by $\tilde{W}_{\text {crit }} / 2$. The inset shows the results for $\tilde{T}=8$

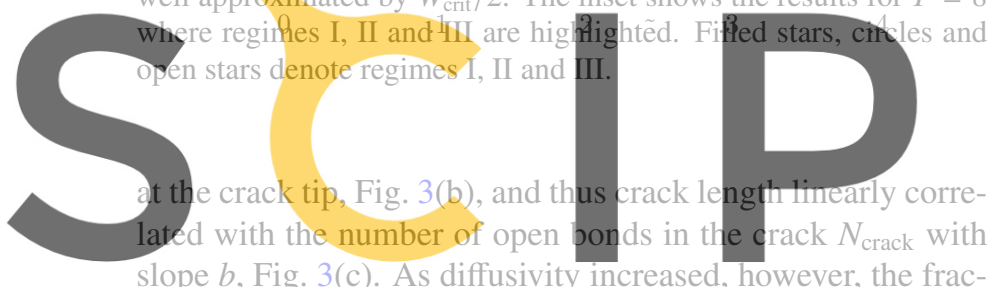

slope b, Fig. 3(c). As diffusivity increased, however, the fraction of failing interfaces increased and the mechanism of fail-

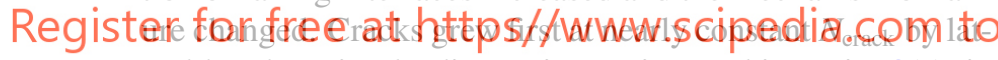
eral bond motion leading to increasing packing, Fig. 3(c), in a behavior reminiscent of observations in cell-cell decohesion [32]. In a second phase, when crowded bonds could not move any further, cracks grew by sequential bond breaking. To explain the higher probability of failure at high $D$, we reasoned that crack growth by biased diffusion of marginal bonds can extend the crack beyond a critical length at which bond breaking dominates rebinding. Thus, our model suggests that mobility of bonds embrittles the adhesive interface, in interesting analogy with the weakening of transient polymer networks by crosslinker mobility [33]. According to this mechanism of embrittlement by bond motion, the progressive immobilization of cadherins as they couple to the cortex observed during maturation of cell-cell junctions should promote stability of the adhesion patch [34].

Following maturation, adhesive cell-cell contacts are known to organize into largely immobilized clusters with $10 \mathrm{~s}$ to 100 s transmembrane adhesion molecules mechanosensitively coupled to the cytoskeleton. The biological and biophysical mechanisms leading to these clusters and their function remain poorly understood [5, 35-37]. However, models of stochastic bond clusters allow us to examine the structural benefit of this organization. To focus on the role of the junctional organization, we considered a sequence of bond archi- tectures shown in Fig. 4(a), where the size of bond clusters and non-adhesive gaps $W_{\mathrm{i}}$ varies but the average bond density and the separation between bonds within clusters remains constant. We studied lifetime of bond clusters of increasing size under fixed pressure and for several tensions by averaging 2000 realizations for each parameter set. In agreement with related studies about the stability of bond clusters attached to an elastic medium [20,21], we found that the lifetime of our hydraulically loaded and tense interfaces changed by orders of magnitude depending on architecture and was largest at intermediates cluster sizes, suggesting a physical selection mechanism and a notion of structural optimality. Furthermore, our results showed that lifetime and optimal cluster size increase with tension, Fig. 4(b). To understand this behavior, we noted that for $W_{\mathrm{i}}<W_{\text {crit }} / 2$ (regime I) the crack size can never be larger than the critical length even when only one bond remains. Consequently lifetime will depend on the competition between binding and unbinding, and in the limit of small clusters, for which rates are uniform and close to $k_{\text {on }}^{0}$ and $k_{\text {off }}^{0}$, lifetime should increase with cluster size [17] in a tensionindependent manner. Our results confirmed these predictions, and showed that the maximum lifetime was attained for cluster sizes closely matching $W_{\text {crit }} / 2$ and thus depending on $P$ and $T$, Fig. 2(c). Focusing then on $W_{i}>W_{\text {crit }}$ (regime III), we

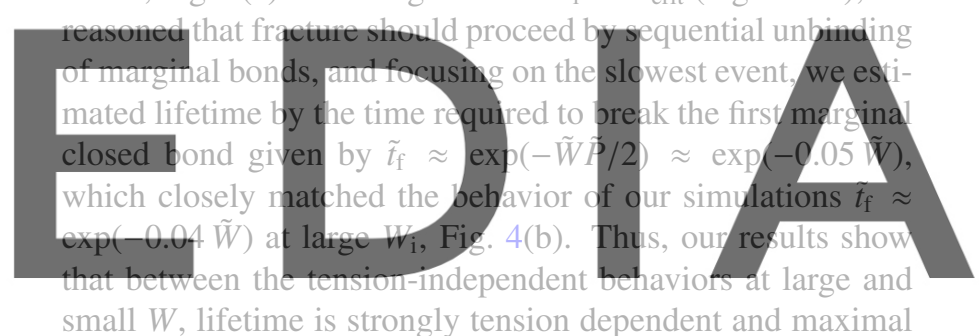

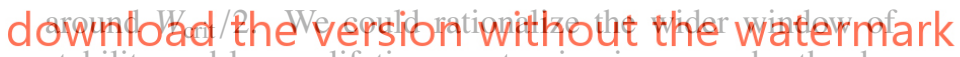
stability and longer lifetimes as tension increases by the dependence of $W_{\text {crit }}$ and $k_{\text {on }}$ on $T$. We finally note that variations of the model studied here showed that the strengthening role of surface tension was not limited to hydraulic loading of the adhesive interface [38].

Taken together, our results show that surface tension strongly enhances the stability of adhesive interfaces loaded hydraulically and bridged by molecular bonds by bringing potential partners closer together and favoring rebinding at the margin of nascent cracks. More specifically, tension controls flaw sensitivity, lifetime and optimal cluster size in such interfaces. We have further established that mobility of molecular bonds embrittles adhesions. Since cells can control surface tension [4, 23] and bonds diffusivity [36], our work identifies a physical mechanism by which cells can dynamically adapt their stickiness, complementary to biochemical mechanisms coupling adhesion and tension [5]. Whether cells and tissues use this physical mechanism to tune adhesion remains to be tested. We speculate that it is compatible with the healing of high tension microlumens at the expense of expanding low tension lumens during early mammalian development [15].

We acknowledge discussions with Alejandro TorresSánchez and the support of the European Research Council (CoG-681434), the European Commission (project 
H2020-FETPROACT-01-2016-731957), and the Generalitat de Catalunya (SGR-1471, ICREA Academia award to MA).

* Email: dimitri.kaurin@upc.edu

Email: marino.arroyo@upc.edu

[1] A. R. Harris, L. Peter, J. Bellis, B. Baum, A. J. Kabla, and G. T. Charras, Proceedings of the National Academy of Sciences 109, 16449 (2012).

[2] L. Casares, R. Vincent, D. Zalvidea, N. Campillo, D. Navajas, M. Arroyo, and X. Trepat, Nat. Mater. 14, 343 (2015).

[3] T. Lecuit and P.-F. Lenne, Nature Reviews Molecular Cell Biology 8, 633 (2007).

[4] C. Guillot and T. Lecuit, Science 340, 1185 (2013).

[5] J.-L. Maître and C.-P. Heisenberg, Curr. Biol. 23, R626 (2013).

[6] A.-S. Smith and E. Sackmann, Chemphyschem 10, 66 (2009).

[7] A. P. Liu and D. A. Fletcher, Nature Reviews Molecular Cell Biology 10, 644 (2009)

[8] E. Sackmann and A.-S. Smith, Soft Matter 10, 1644 (2014).

[9] D. Schmidt, T. Bihr, S. Fenz, R. Merkel, U. Seifert, K. Sengupta, and A. S. Smith, Biochimica et Biophysica Acta Molecular Cell Research 1853, 2984 (2015).

[10] E. M. Schmid, M. H. Bakalar, K. Choudhuri, J. Weichsel, H. S. Ann, P. L. Geissler, M. L. Dustin, and D. A. Fletcher, Nat. Phys. 12, 704 (2016).

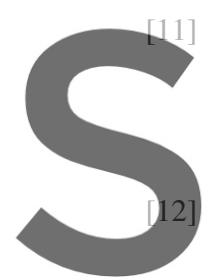
A. J. Kosmalska, L. Casare tacherry, R. Moreno-Vicente, Pozo, S. Mayor, M. Arroyo, I thier, and P. Roca-Cusachs, EP (2015)

E. Latorre, S. Kale, L. Casare L. Valon, R. V. Nair, E. Garre et al., Nature 563, 203 (2018)

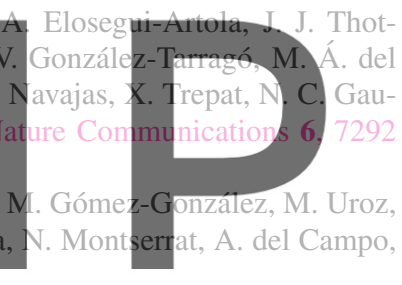

[13] S. Sigurbjörnsdóttir, R. Mathew, and M. Leptin, Nature Re-

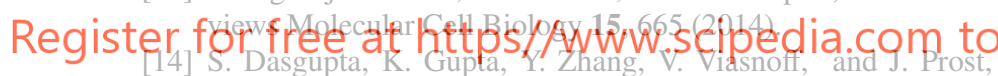
Proceedings of the National Academy of Sciences 115, E4751 (2018)

[15] J. G. Dumortier, M. Le Verge-Serandour, A. F. Tortorelli, A. Mielke, L. de Plater, H. Turlier, and J.-L. Maître, Science 365, 465 (2019).

[16] G. I. Bell, Science 200, 618 (1978).

[17] T. Erdmann and U. S. Schwarz, The Journal of chemical physics
121, 8997 (2004).

[18] E. Reister-Gottfried, K. Sengupta, B. Lorz, E. Sackmann, U. Seifert, and A. S. Smith, Physical Review Letters 101, 1 (2008).

[19] H. Krobath, B. Rózycki, R. Lipowsky, and T. R. Weikl, PLoS ONE 6 (2011).

[20] J. Qian, J. Wang, and H. Gao, Langmuir 24, 1262 (2008).

[21] J. Qian, J. Wang, Y. Lin, and H. Gao, Biophysical Journal 97, 2438 (2009).

[22] Y. Mulla, G. Oliveri, J. T. Overvelde, and G. H. Koenderink, Physical review letters 120, 268002 (2018).

[23] P. Sens and M. S. Turner, Physical Review E 73, 031918 (2006).

[24] See Supplemental Material at [URL will be inserted by publisher] for additional information on the theoretical model, the computational algorithms and for videos illustrating the fracture behavior.

[25] T. Erdmann and U. S. Schwarz, The European Physical Journal E: Soft Matter and Biological Physics 22, 123 (2007).

[26] D. T. Gillespie, The journal of physical chemistry 81, 2340 (1977).

[27] A. T. Zehnder, Fracture Mechanics (Springer Netherlands, 2012).

[28] G. Bell, M. Dembo, and P. Bongrand, Biophysical Journal 45. 1051 (1984)

[29] M. Arroyo and X. Trepat, Science 365, 442 (2019).

[30] F. Brochard-Wyart and P.-G. de Gennes, Proc. Natl. Acad. Sci. U. S. A. 99, 7854 (2002).

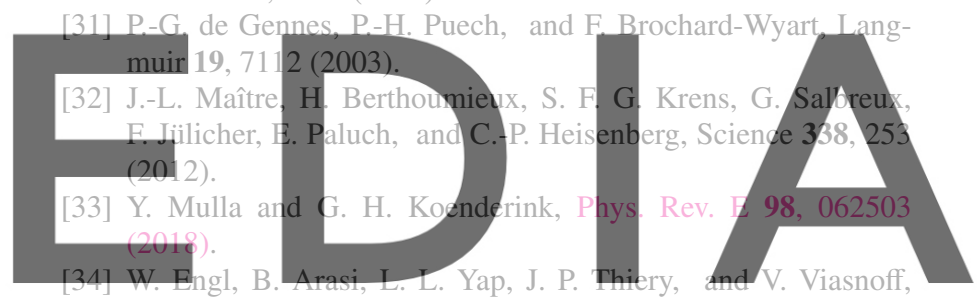

Nature Cell Biology 16, 584 (2014).

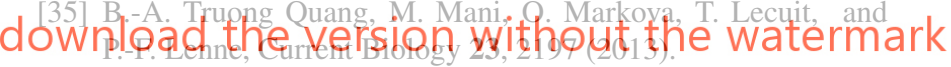

[36] A. S. Yap, G. A. Gomez, and R. G. Parton, Developmental Cell 35,12 (2015).

[37] R. Changede and M. Sheetz, BioEssays 39, 1 (2017).

[38] D. Kaurin, A theoretical and computational study of soft adhesion mediated by specific binders, Ph.D. thesis, Universitat Politècnica de Catalunya (2018). 